\title{
Using Size-Exclusion Chromatography to Monitor Variations in the Sizes of Microwave-Irradiated Gold Nanoparticles
}

\author{
Fu-Ken Liu \\ Department of Applied Chemistry, National University of Kaohsiung, No. 700 Kaohsiung University Road, \\ Nan Tzu District, Kaohsiung 811, Taiwan \\ Correspondence should be addressed to Fu-Ken Liu, fkliu@nuk.edu.tw
}

Received 13 November 2012; Accepted 30 November 2012

Academic Editors: M. C. Bruzzoniti, L. Chen, J. Matysiak, and L. Membrado

Copyright $\odot 2012$ Fu-Ken Liu. This is an open access article distributed under the Creative Commons Attribution License, which permits unrestricted use, distribution, and reproduction in any medium, provided the original work is properly cited.

\begin{abstract}
Size-exclusion chromatography (SEC) was used to evaluate gold nanoparticles (Au NPs) for variations in their sizes after microwave (MW) irradiation, with the eluted NPs monitored through diode array detection to reveal their surface plasmon absorptions. The sizes of citrate-capped Au NPs decreased upon increasing the MW irradiation temperature, consistent with digestive ripening of these NPs under the operating conditions. In contrast, Au NPs capped with sodium dodecyl sulfate increased in size upon increasing the MW irradiation temperature, consistent with Ostwald ripening. When the Au NPs were capped with 3A-amino$3 \mathrm{~A}$-deoxy-(2AS,3AS)- $\beta$-cyclodextrin $\left(\mathrm{H}_{2} \mathrm{~N}-\beta-\mathrm{CD}\right)$, however, their dimensions were barely affected by the MW irradiation temperature, confirming that $\mathrm{H}_{2} \mathrm{~N}-\beta-\mathrm{CD}$ is a good stabilizer against MW irradiation. Therefore, SEC-with its short analysis times, low operating costs, automated operation, and in situ analysis—-has great potential for use in the rapid monitoring of NPs subjected to treatment under various MW irradiation conditions.
\end{abstract}

\section{Introduction}

Metal nanoparticles (NPs) are attracting a great deal of attention from practitioners in a wide variety of scientific fields [1-13] because their physical and chemical properties are related to their chemical compositions, sizes, and surface structural characteristics [14-17]. Among all metal NPs, Au NPs are especially attractive for research in nanotechnology [18], for example, in the detection of cancer [19], DNA [20], and even single molecules through surface-enhanced Raman spectroscopy $[21,22]$. Because the chemical or physical properties of Au NPs are size dependent [9, 23, 24], monitoring the sizes of Au NPs is a critical step toward understanding how their functions are related to their dimensions $[25,26]$.

Several research articles have reported the reaction kinetics of Au NP formation using the citrate method [27$31]$. Both Ostwald ripening [28, 29], whereby particles increase in size during the reaction, and digestive ripening, where the average particle size decreases, have been observed $[30,31]$ — with the latter being used to break colloids into smaller particles through the addition of ligands. As a result, many strategies have been developed for varying the sizes of $\mathrm{Au}$ NPs [25]. Herein, we wished to establish a rapid and efficient strategy for determining the size-dependent chemical or physical properties of Au NPs prepared using a chosen treatment method.

Many methods are currently available for particle size analysis [32], including transmission electron microscopy (TEM), dynamic light scattering (DLS), analytical ultracentrifugation (AUC), asymmetric flow field flow fractionation (aF-FFF), X-ray diffraction (XRD), and UV-Vis spectroscopy. In addition, techniques that can be performed using a small volume of a single sample within a short analysis times and at a low operating cost are of interest for the separation NPs into their constituent sizes [33]. Relative to other separation techniques (e.g., centrifugation, high-performance liquid chromatography (HPLC) [9], capillary electrophoresis [34], diafiltration [35], and gel electrophoresis [36]), size-exclusion chromatography (SEC) has the advantageous features of short analysis times, low operating costs, and high reliability, with the potential for in situ analysis and in situ gathering of spectroscopic data of the individual separated NPs [37]. 
In previous studies, we demonstrated that SEC analysis could be applied as a stand-alone technique to determine the sizes of synthetic $\mathrm{Au}, \mathrm{Au} / \mathrm{Pd}$ core/shell, and $\mathrm{Au} / \mathrm{Pt}$ core/shell NPs at room temperature [33, 38, 39]. In addition, we have recently employed SEC approaches to determine a suitable stabilizer for NPs under conditions mimicking the temperatures found in catalysis environments $\left(\mathrm{ca} .100^{\circ} \mathrm{C}\right.$ ) [37] and to rapidly monitor the sizes of Au NPs in the presence of salt or organic environments to determine the effects of the stabilizers of Au NPs in these media [40]. Our results, combined with the prevalence of automated SEC systems, suggest that this approach is superior to other techniques (e.g., DLS and TEM) typically employed for the characterization of NPs [33].

In this study, we monitored the variations in size of $\mathrm{Au}$ NPs in the presence of different capping agents-citrate, sodium dodecyl sulfate (SDS), and 3A-amino-3A-deoxy(2AS,3AS)- $\beta$-cyclodextrin $\left(\mathrm{H}_{2} \mathrm{~N}-\beta\right.$-CD) - under microwave (MW) irradiation treatment at desired temperatures. In contrast to our previous studies, all of the experiments described herein were based on MW irradiation treatment at temperatures greater than $100^{\circ} \mathrm{C}$. We selected MW irradiation as a variable in this study because it is an efficient heating method that allows precise temperature control as a built-in function in commercial MW instruments. As a result, MW irradiation was a convenient tool for studying the relationship between thermal effects and the size of NPs, especially for reaction temperatures of greater than $100^{\circ} \mathrm{C}$-difficult to achieve using conventional hot-plate heating methods [41]. To the best of our knowledge, no previous reports have described the feasibility of using SEC methods to monitor the sizes of Au NPs under MW irradiation conditions. Through this study, we have found that SEC is highly applicable to the rapid analysis of MW-treated Au NPs.

\section{Materials and Methods}

2.1. Materials. Hydrogen tetrachloroaurate $\left(\mathrm{HAuCl}_{4}\right)$ was purchased from Acros Organics (Geel, Belgium). Sodium citrate was obtained from Merck (Darmstadt, Germany). SDS and $\mathrm{H}_{2} \mathrm{~N}-\beta-\mathrm{CD}$ were obtained from Tokyo Chemical Industry (Tokyo, Japan). All eluents were prepared afresh each day and filtered through a $0.45-\mu \mathrm{m}$ membrane filter (Alltech, Deerfield, IL, USA) prior to use. Deionized water

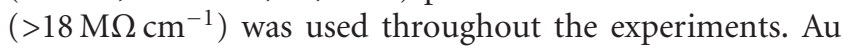
NPs having a mean diameter of $11.5 \pm 0.6 \mathrm{~nm}$ were homemade; their fabrication is described in the following section. Standard Au NPs having a mean diameter of $21.3 \pm 1.3 \mathrm{~nm}$ were obtained from Sigma (St. Louis, MO, USA). Au NPs having mean diameters of $40.1 \pm 3.2$ and $59.9 \pm 4.8 \mathrm{~nm}$ were obtained from BBInternational (Cardiff, UK).

2.2. Instrumentation. Different capping agents were placed in $\mathrm{Au}$ NP solutions that were then treated with an SP-D MW irradiation system (CEM, Matthews, NC, USA). The $\mathrm{MW}$ system was operated at powers in the range from 0 to $200 \mathrm{~W}$; samples were treated with MW irradiation for $20 \mathrm{~min}$ with stirring at desired temperatures. An L-2000 liquid chromatograph (Hitachi, Tokyo, Japan) was used for SEC separation of the Au NPs. The SEC apparatus was equipped with a diode array detection (DAD) system. A Nucleogel GFC 60-8 column (Macherey-Nagel, Duren, Germany; $300 \times$ $7.7 \mathrm{~mm}$; pore size: $4000 \mathrm{~nm}$; particle size: $8 \mu \mathrm{m}$ ) and a 0.5 $\mu \mathrm{m}$ precolumn filter were employed for analytical separation;

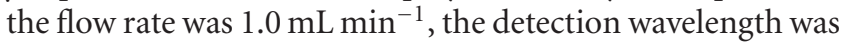
$520 \mathrm{~nm}$, and the injection volume was $20 \mu \mathrm{L}$. A JEOL JEM2010 transmission electron microscope (Tokyo, Japan) was used to characterize the sizes of the Au NPs.

2.3. Synthesis of $A u$ NPs. The synthetic procedure for the formation of $\mathrm{Au}$ NPs through citrate-mediated reduction of $\mathrm{HAuCl}_{4}$ has been described elsewhere [42]. The sizes of the particles in the sample were determined through twodimensional (2D) grain analysis after digitizing TEM images. The average particle diameter of the synthesized Au NPs was estimated to be $11.5 \pm 0.6 \mathrm{~nm}$.

\section{Result and Discussion}

3.1. Separation of NPs Using SEC. Au NPs of four different sizes $(11.5 \pm 0.6,21.3 \pm 1.3,40.1 \pm 3.2$, and $59.9 \pm 4.8 \mathrm{~nm})$ were separated in the presence of a mobile phase additive (10 mM SDS) at a flow rate of $1.0 \mathrm{~mL} \mathrm{~min}^{-1}$. Accordingly, we found that the four differently sized Au NPs had different elution times; the sequence of elution times (increasing with decreasing Au NP size) paralleled the elution behavior described previously [37]. For SEC to be used practically for the routine analysis of NP sizes, it was necessary for us to validate the reproducibility of the elution times because it influences the precision of the size-characterization process. Thus, we measured the relative standard deviations of the elution times from five consecutive runs using the 11.5-, 21.3-, 40.1-, and 59.9-nm diameter Au NP standards; the calculated precisions were $0.10,0.05,0.13$, and $0.28 \%$, respectively; that is, the elution behavior of Au NPs through SEC was highly reproducible.

Figure 1 indicates that a strong correlation $\left(R^{2}=0.996\right)$ existed between the elution times and the diameters of the NPs; the error bars in the $y$ - and $x$-dimensions represent the variations of the elution times and diameters, respectively, in terms of one standard deviation. This plot confirms that it is possible to employ SEC to characterize NPs having sizes in the nanometer regime.
3.2. Effect of $M W$ Irradiation Treatment Temperature on Sizes of Citrate-Capped Au NPs. In these experiments, we obtained Au NP solutions after the addition of sodium citrate solution to a heated solution of Au precursor ions. During the synthesis of the Au NPs, the citrate ions performed several tasks; they complexed strongly to the Au metal ions, reduced them, and capped the resulting Au NPs. To examine the size variations of the citrate-capped $\mathrm{Au}$ NPs under MW irradiation treatment, we placed Au NPs (fabricated as described in Materials and Methods, $0.5 \mathrm{~mL}$ ) into three MW reaction vials $(10 \mathrm{~mL})$, added deionized water $(0.5 \mathrm{~mL})$ to each vial, and then sealed the vials and treated them at 100, 120 , or $140^{\circ} \mathrm{C}$ in a MW irradiation system with stirring for approximately $20 \mathrm{~min}$. After cooling to room temperature, we performed SEC analysis of the Au NPs. 


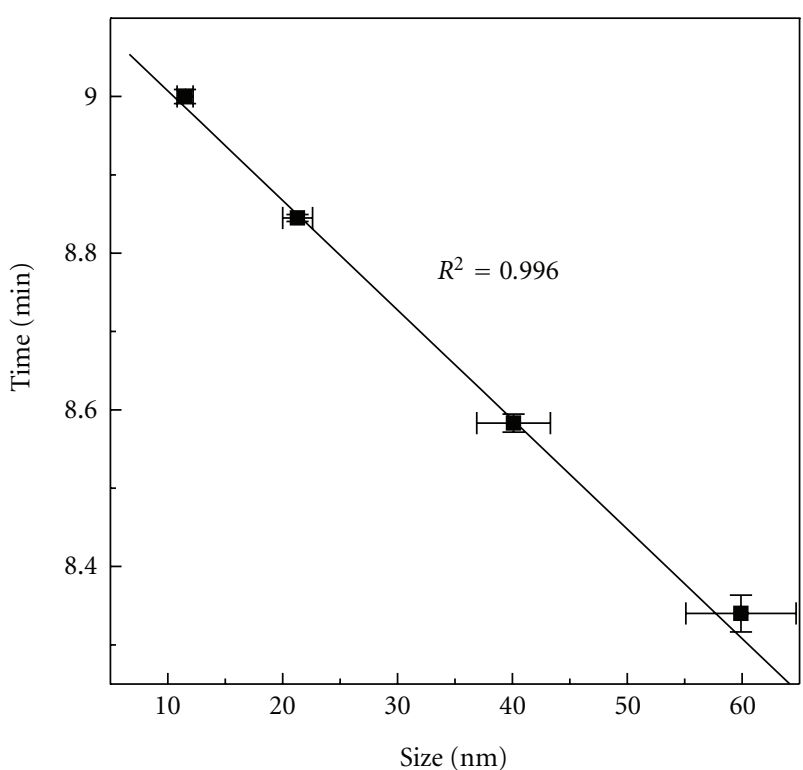

FIGURE 1: Calibration curve for the elution times of Au NPs plotted with respect to their diameters. The error bars in the $y$ - and $x$-dimensions represent the variations of the elution times and diameters, respectively, in terms of one standard deviation. The separation conditions are described in Section 2.

Figure 2(a) displays chromatograms obtained from the solutions of the citrated-capped Au NPs that had been subjected to MW irradiation treatment at 100, 120, and $140^{\circ} \mathrm{C}$. In each chromatogram, the intensity of the signal for the Au NPs decreased. In addition, the retention time lengthened slightly upon increasing the heating temperaturepresumably a result of digestive ripening, which decreases the sizes of citrate-capped Au NPs [25, 37]. We used the calibration curve in Figure 1 to obtain the sizes of the fabricated NPs from the elution times in the SEC chromatograms of the NPs. Accordingly, the SEC chromatograms in Figure 2(a) revealed that the $\mathrm{Au}$ NPs that were eluted from the MW samples treated at 100,120 , and $140^{\circ} \mathrm{C}$ had diameters of 7.7, 7.5, and $4.1 \mathrm{~nm}$, respectively. Thus, SEC analysis revealed that the sizes of the citrate-capped Au NPs decreased upon increasing the MW irradiation temperature, consistent with digestive ripening of the Au NPs occurring during the MW irradiation treatment processes. The phenomenon of digestive ripening of Au NPs has also been observed previously for citratecapped Au NPs after prolonged photoirradiation [25].

Evidence for the digestive ripening of citrate-capped Au NPs after MW irradiation was also apparent from the eluted samples' surface plasmon (SP) absorptions in the UV-Vis absorption spectra obtained using the DAD system (Figure 2(b)). Link and El-Sayed reported that the sizes of Au NPs are related to the values of $\lambda_{\max }$ of their SP absorptions [43], which are blue shifted upon decreasing the sizes of $\mathrm{Au}$ NPs. Therefore, the use of SEC combined with a DAD system provides added value in terms of revealing the spectroscopic properties of the individual sets of separated Au NPs. Herein, we also used Au NPs' SP absorptions in Figure 2(b) to monitor their variations in particle diameters.
The corresponding values of $\lambda_{\max }$ of the SP absorptions of the $\mathrm{Au}$ NPs that had been subjected to MW irradiation treatment at 100,120 , and $140^{\circ} \mathrm{C}$ were 522,521 , and $520 \mathrm{~nm}$, respectively, consistent with the sizes of the citrate-capped Au NPs having decreased slightly (i.e., digestive ripening occurred to form smaller particles) upon increasing the MW irradiation treatment temperature.

3.3. Effect of MW Heating Treatment Temperature on Sizes of SDS-Capped Au NPs. Surfactants can be employed as stabilizers in the size-selective preparation of metal particles $[44,45]$, with the ionic units surrounding the metal cores to prevent their agglomeration. Those previous studies, however, focused on the effects of surfactants in NP reaction media at temperatures lower than $40^{\circ} \mathrm{C}$. In contrast, in this present study, we were interested in discovering whether the use of SDS as an additive anionic surfactant in the sample matrix would influence the sizes of Au NPs under MW irradiation treatment at high temperatures $\left(>100^{\circ} \mathrm{C}\right)$. To examine the variations in size of SDS-capped Au NPs under MW irradiation, we placed Au NPs (fabricated as described in Materials and Methods, $0.5 \mathrm{~mL}$ ) into three $\mathrm{MW}$ reaction vials $(10 \mathrm{~mL})$, added deionized water $(0.4 \mathrm{~mL})$ and SDS $(100 \mathrm{mM}$, $0.1 \mathrm{~mL}$ ) to each vial, and then sealed the vials and subjected them to MW irradiation treatment at 100,120 , or $140^{\circ} \mathrm{C}$ with stirring for approximately $20 \mathrm{~min}$. After cooling to room temperature, we performed SEC analysis of the Au NPs.

Figure 3(a) displays SEC chromatograms of the SDScapped Au NPs samples that had been subjected to MW irradiation treatment at temperatures of 100,120 , and $140^{\circ} \mathrm{C}$ for $20 \mathrm{~min}$. The intensity of the signal for the Au NPs decreased upon increasing the heating temperature; in addition, the elution time for the Au NPs decreased accordinglypresumably as a result of Ostwald ripening, which increases the sizes of $\mathrm{Au}$ NPs $[25,37]$. The elution times in the SEC chromatograms of the NPs in Figure 3(a) suggested that the eluted NPs from samples treated with MW irradiation at 100,120 , and $140^{\circ} \mathrm{C}$ had diameters of 10.5 , 14.8 , and $24.8 \mathrm{~nm}$, respectively. These findings from SEC analysis revealed that the sizes of the SDS-capped Au NPs increased upon increasing the MW irradiation temperature, confirming that Ostwald ripening was occurring under these conditions. The presence of SDS in the reaction medium might be an important factor for nanomaterials to express Ostwald ripening phenomena; Wang et al. reported a similar SDS-induced Ostwald ripening in the formation of hollow $\mathrm{CaWO}_{4}$ microspheres [46].

Evidence for Ostwald ripening of the SDS-capped $\mathrm{Au}$ NPs during the MW irradiation processes was also apparent from the SP absorptions of the eluted samples in the UVVis absorption spectra obtained using the DAD system (Figure 3(b)). Because the sizes of Au NPs can be monitored from their SP absorptions [43], we used the Au NPs' SP absorptions in Figure 3(b) to monitor the variations of their particle diameters.

The values of $\lambda_{\max }$ of the SP absorptions of the SDScapped Au NPs that had been subjected to MW irradiation treatment at 100,120 , and $140^{\circ} \mathrm{C}$ were 522,523 , and $528 \mathrm{~nm}$, respectively. These results follow the same trend obtained 


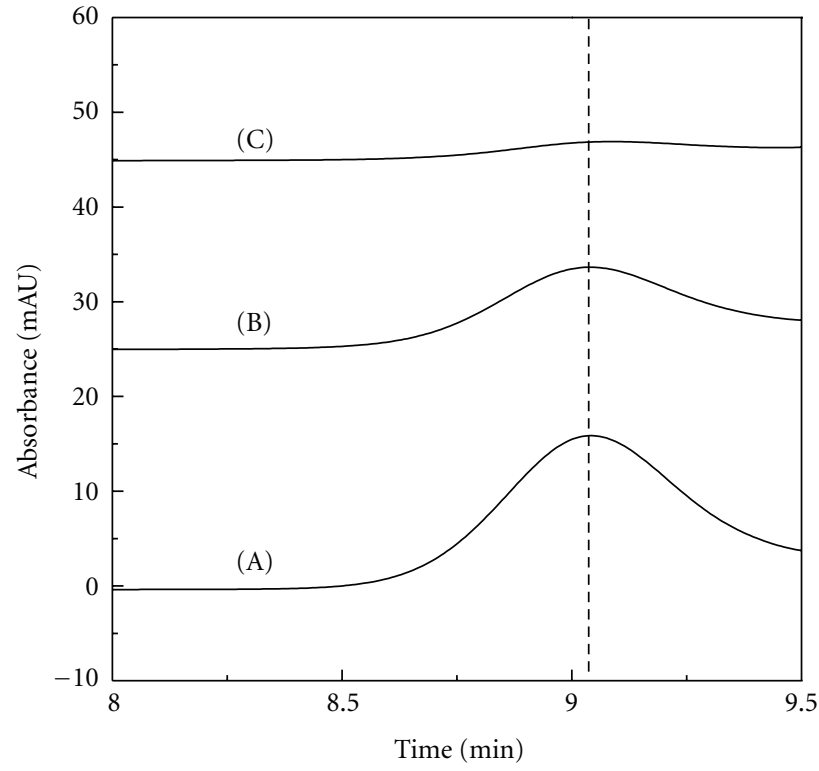

(a)

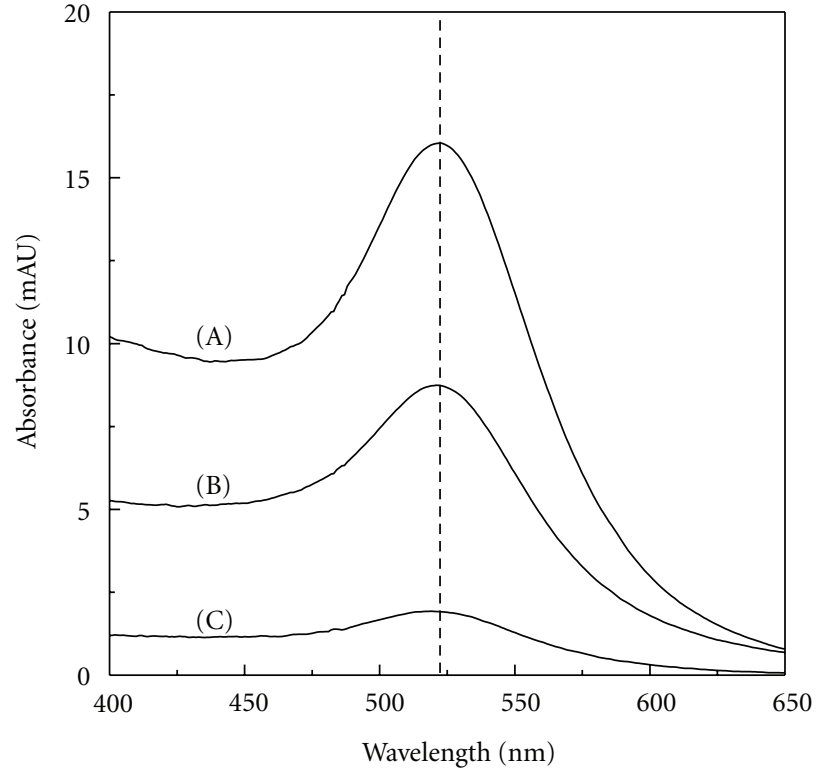

(b)

FIgURE 2: (a) SEC chromatograms and (b) UV-Vis absorption spectra of citrate-capped Au NPs after MW irradiation at temperatures of (A) 100, (B) 120 , and (C) $140^{\circ} \mathrm{C}$. The separation conditions are described in Section 2.

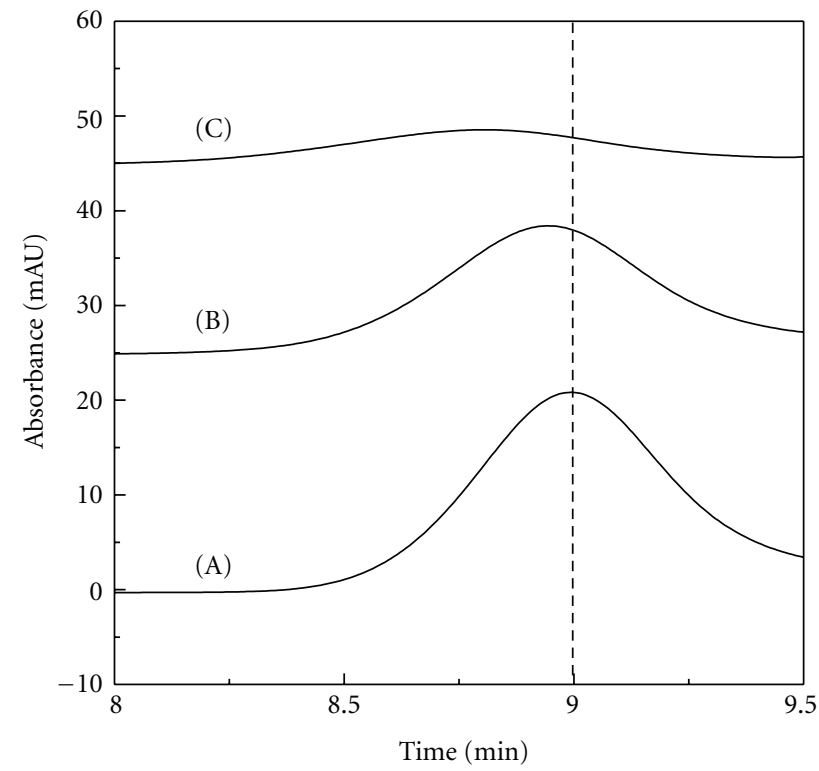

(a)

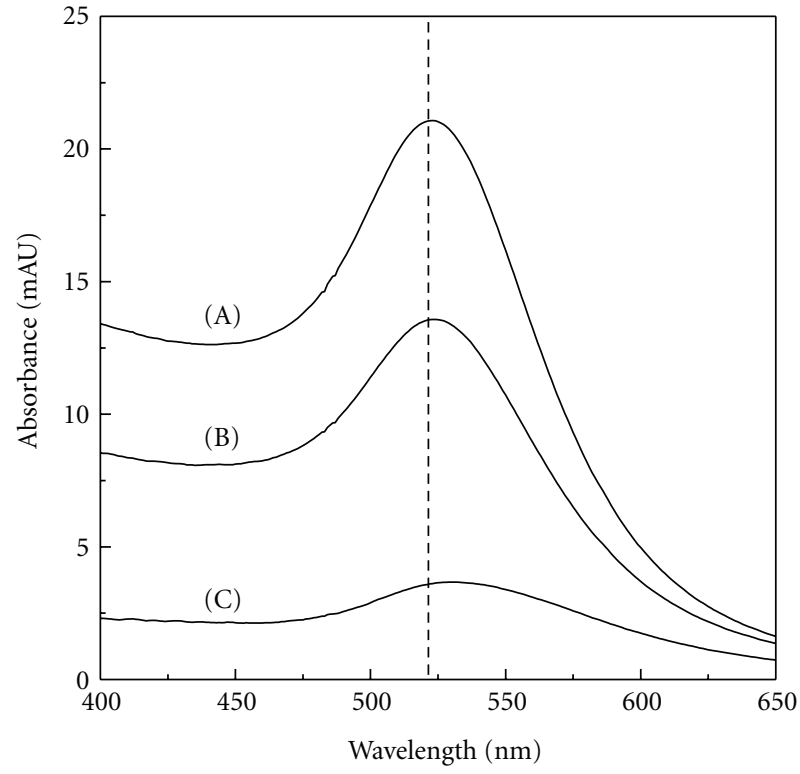

(b)

FIGURE 3: (a) SEC chromatograms and (b) UV-Vis absorption spectra of SDS-capped Au NPs after MW irradiation at temperatures of (A) 100, (B) 120 , and (C) $140^{\circ} \mathrm{C}$. The separation conditions are described in Section 2.

in Figure 3(a); that is, the sizes of the SDS-capped Au NPs increased upon increasing the MW irradiation treatment temperature. Therefore, our findings confirmed that the SDS-capped Au NPs did increase slightly (i.e., Ostwald ripening occurred to form larger particles) upon increasing the MW irradiation treatment temperature.

3.4. Effect of MW Heating Treatment Temperature on Sizes of $\mathrm{H}_{2} \mathrm{~N}-\beta$-CD-Capped Au NPs. Employing cyclodextrins (CDs) as capping agents for Au NPs, Liu et al. recently reported [4749] that surface-attachment of CDs imparted Au NPs with excellent solubility in aqueous media. Furthermore, TEM analysis verified that these CD-capped Au NPs were well dispersed [49]; in addition, they exhibited excellent catalytic activity [50]. These features indicate that CDs have strong stabilizing effects on NPs, preventing them from aggregation, and, therefore, allowing them to function as usable materials for catalytic applications. In addition, we have also previously 


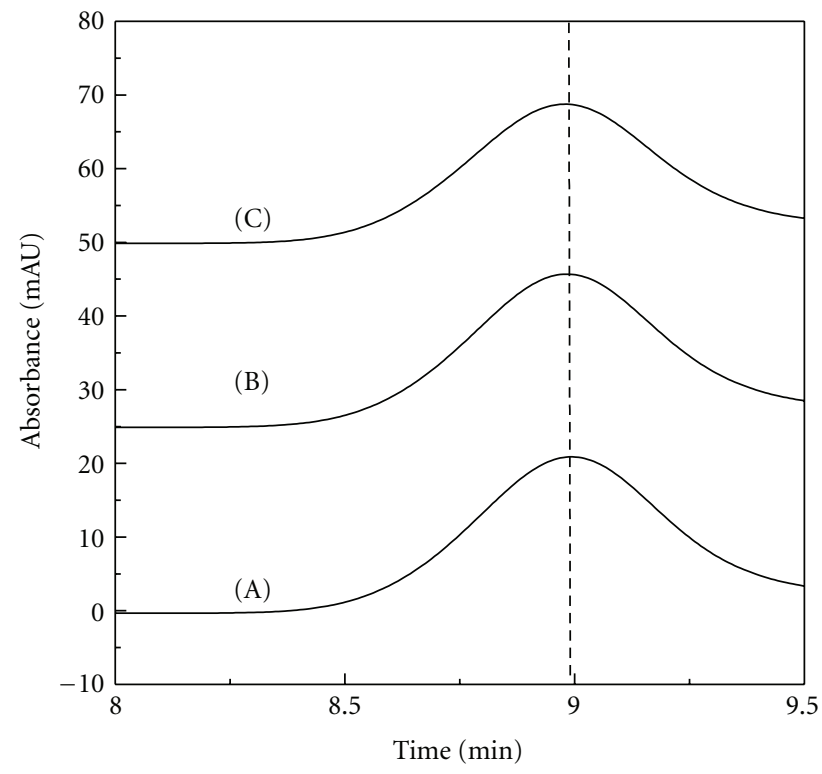

(a)

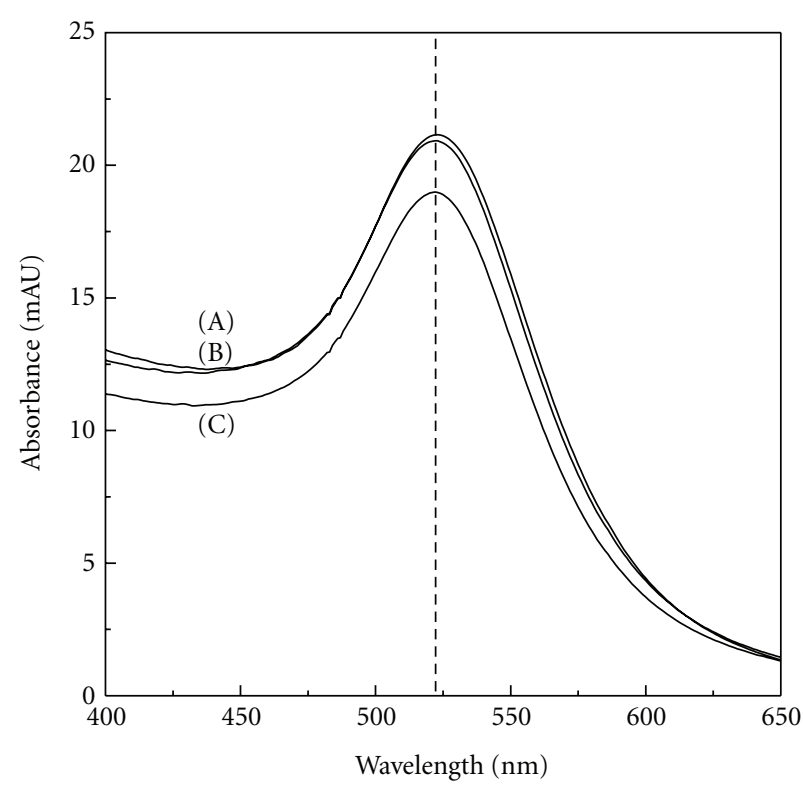

(b)

Figure 4: (a) SEC chromatograms and (b) UV-Vis absorption spectra of $\mathrm{H}_{2} \mathrm{~N}$ - $\beta$-CD-capped Au NPs after MW irradiation at temperatures of (A) 100, (B) 120 , and (C) $140^{\circ} \mathrm{C}$. The separation conditions are described in Section 2.

found that Au NPs capped with an amino derivative of $\beta$ $\mathrm{CD}\left(\mathrm{H}_{2} \mathrm{~N}-\beta-\mathrm{CD}\right)$ have good thermal stability when treated in a thermal oven $\left(\mathrm{ca} .100^{\circ} \mathrm{C}\right)$ [37]. In this present study, we wished to test whether $\mathrm{H}_{2} \mathrm{~N}-\beta-\mathrm{CD}$, as an additive in the $\mathrm{Au}$ NP medium, would result in any variation in size of $\mathrm{Au}$ NPs subjected to MW irradiation treatment at temperatures beyond $100^{\circ} \mathrm{C}$.

To examine the behavior of $\mathrm{H}_{2} \mathrm{~N}-\beta$-CD-capped Au NPs under MW irradiation at different temperatures, we placed Au NPs $(0.5 \mathrm{~mL})$ into three MW reaction vials $(10 \mathrm{~mL})$, added deionized water $(0.4 \mathrm{~mL})$, and $\mathrm{H}_{2} \mathrm{~N}-\beta-\mathrm{CD}(100 \mathrm{mM}$, $0.1 \mathrm{~mL}$ ) to each vial, and then sealed the vials and subjected them to MW irradiation treatment at 100,120 , or $140^{\circ} \mathrm{C}$ with stirring for approximately $20 \mathrm{~min}$. After cooling to room temperature, we performed SEC analysis of the Au NPs.

Figure 4(a) displays the chromatograms obtained after MW irradiation of the $\mathrm{H}_{2} \mathrm{~N}-\beta$-CD-capped Au NPs. The elution times in the chromatograms of these three samples were very similar. From the calibration curve in Figure 1, the SEC chromatograms in Figure 4(a) sets A, B, and C indicate that the samples of $\mathrm{H}_{2} \mathrm{~N}-\beta$-CD-capped Au NPs all had an average diameter of $11.3 \mathrm{~nm}$. Thus, SEC analysis revealed that the sizes of the $\mathrm{H}_{2} \mathrm{~N}-\beta$-CD-capped Au NPs were very similar after MW irradiation at different temperatures.

During the separations of the Au NPs, we also recorded the SP absorptions of the eluted samples. Figure 4 (b) presents the corresponding SP absorptions of the $\mathrm{H}_{2} \mathrm{~N}-\beta-\mathrm{CD}$-capped $\mathrm{Au}$ NPs after sampling the chromatograms in Figure 4(a) at their maximum signal intensities (each at an elution time of ca. $9.00 \mathrm{~min}$ ). All of the SP absorption peaks had a value of $\lambda_{\max }$ of approximately $522 \mathrm{~nm}$, indicating that the sizes of the Au NPs did not change significantly in this medium, regardless of the MW irradiation temperature. Taken together, the results in Figures 4(a) and 4(b) suggest that $\mathrm{H}_{2} \mathrm{~N}-\beta-\mathrm{CD}$ is a good stabilizer for Au NPs against MW irradiation, even at treatment temperatures greater than $100^{\circ} \mathrm{C}$; in addition, this stability is superior to that of $\mathrm{Au} \mathrm{NP}$ suspensions in citrate or SDS (cf. Figures 2 and 3 ).

The mechanism through which $\mathrm{H}_{2} \mathrm{~N}-\beta$-CD stabilizes $\mathrm{Au}$ NPs most likely involves strong association of the Au NP surfaces with the amino groups [51], presumably through interactions that limit the size variation of the Au NPs [52]. Therefore, the $\mathrm{H}_{2} \mathrm{~N}-\beta$-CD-capped Au NPs were stable under MW irradiation.

Because the process of characterizing the analytes took less than $12 \mathrm{~min}$ (see Figures 2(a), 3(a), and 4(a)), SEC appears to be a good tool for rapidly determining suitable capping agents for varying the sizes of Au NPs under MW irradiation. We believe that the mechanisms through which the Au NPs associate with these and other protecting agents could be determined readily through the use of common, modern, automated SEC systems.

\section{Conclusion}

We have employed citrate-mediated reduction to produce $\mathrm{Au}$ NP solutions that we then subjected to MW irradiation at desired temperatures - using either the as-prepared medium (citrate capped) or SDS- or $\mathrm{H}_{2} \mathrm{~N}-\beta$-CD-capped Au NPs. We then monitored the resulting solutions through SEC analysis and UV-Vis absorption spectroscopy of the eluted Au NPs, noting that different capping agents affected the sizes of the Au NPs to different degrees. For the citrate-capped Au NPs, the average diameter decreased upon increasing the MW irradiation temperature, consistent with digestive ripening of the Au NPs under the operating conditions. The average 
size of the SDS-capped Au NPs increased upon increasing the MW irradiation temperature, consistent with Ostwald ripening of the Au NPs. When the Au NPs were capped with $\mathrm{H}_{2} \mathrm{~N}-\beta$-CD units, the average dimensions of the Au NPs were barely affected by the MW treatment, revealing that $\mathrm{H}_{2} \mathrm{~N}-\beta$ $\mathrm{CD}$ is a good stabilizer for Au NPs against MW irradiation.

Our results suggest that SEC has some major benefits enabling the monitoring of Au NPs; for example, it might be useful for rapidly ( $<12 \mathrm{~min}$ ) characterizing the size manipulation of Au NPs to ensure that they reach their optimal chemical or physical properties. SEC also aids in the search for new capping agents to control the sizes of Au NP products. In addition, when using SEC in conjunction with a DAD system, we can also obtain added value in terms of the spectroscopic properties of the individual sets of separated Au NPs.

\section{Acknowledgment}

This study was supported financially by the National Science Council, Taiwan (NSC 100-2113-M-390-002-MY3).

\section{References}

[1] F. K. Liu, "Analysis and applications of nanoparticles in the separation sciences: a case of gold nanoparticles," Journal of Chromatography A, vol. 1216, no. 52, pp. 9034-9047, 2009.

[2] N. R. Jana, L. Gearheart, and C. J. Murphy, "Evidence for seedmediated nucleation in the chemical reduction of gold salts to gold nanoparticles," Chemistry of Materials, vol. 13, no. 7, pp. 2313-2322, 2001.

[3] G. Schmid, Clusters and Colloids, from Theory to Applications, Wiley-VCH, New York, NY, USA, 1994.

[4] G. Schmid, M. Bäumle, M. Geerkens, I. Heim, C. Osemann, and T. Sawitowski, "Current and future applications of nanoclusters," Chemical Society Reviews, vol. 28, no. 3, pp. 179-185, 1999.

[5] J. D. Aiken and R. G. Finke, "A review of modern transitionmetal nanoclusters: their synthesis, characterization, and applications in catalysis," Journal of Molecular Catalysis A, vol. 145, no. 1-2, pp. 1-44, 1999.

[6] C. N. R. Rao, G. U. Kulkarni, P. J. Thomas, and P. P. Edwards, "Metal nanoparticles and their assemblies," Chemical Society Reviews, vol. 29, no. 1, pp. 27-35, 2000.

[7] A. C. Templeton, W. P. Wuelfing, and R. W. Murray, "Monolayer-protected cluster molecules," Accounts of Chemical Research, vol. 33, no. 1, pp. 27-36, 2000.

[8] A. Henglein, "Small-particle research: physicochemical properties of extremely small colloidal metal and semiconductor particles," Chemical Reviews, vol. 89, no. 8, pp. 1861-1873, 1989.

[9] A. Henglein, "Physicochemical properties of small metal particles in solution: "Microelectrode" reactions, chemisorption, composite metal particles, and the atom-to-metal transition," Journal of Physical Chemistry, vol. 97, no. 21, pp. 5457-5471, 1993.

[10] S. W. Chen, R. S. Ingram, M. J. Hostetler et al., "Gold nanoelectrodes of varied size: transition to molecule-like charging," Science, vol. 280, no. 5372, pp. 2098-2101, 1998.

[11] N. R. Jana, T. K. Sau, and T. Pal, "Growing small silver particle as redox catalyst," Journal of Physical Chemistry B, vol. 103, no. 1, pp. 115-121, 1999.
[12] C. J. Murphy, "Nanocubes and nanoboxes," Science, vol. 298, no. 5601, pp. 2139-2141, 2002.

[13] T. H. Chang, F. K. Liu, Y. C. Chang, and T. C. Chu, "Rapidly characterizing the growth of Au nanoparticles by CE," Chromatographia, vol. 67, no. 9-10, pp. 723-730, 2008.

[14] G. T. Wei and F. K. Liu, "Separation of nanometer gold particles by size exclusion chromatography," Journal of Chromatography A, vol. 836, no. 2, pp. 253-260, 1999.

[15] C. A. Mirkin, R. L. Letsinger, R. C. Mucic, and J. J. Storhoff, "A DNA-based method for rationally assembling nanoparticles into macroscopic materials," Nature, vol. 382, no. 6592, pp. 607-609, 1996.

[16] A. Taleb, C. Petit, and M. P. Pileni, "Synthesis of highly monodisperse silver nanoparticles from AOT reverse micelles: a way to 2D and 3D self-organization," Chemistry of Materials, vol. 9, no. 4, pp. 950-959, 1997.

[17] J. A. Ascencio, H. B. Liu, U. Pal, A. Medina, and Z. L. Wang, "Transmission electron microscopy and theoretical analysis of $\mathrm{AuCu}$ nanoparticles: atomic distribution and dynamic behavior," Microscopy Research and Technique, vol. 69, no. 7, pp. 522-530, 2006.

[18] K. B. Male, J. Li, C. C. Buh, S. C. Ng, and J. H. T. Luong, "Synthesis and stability of fluorescent gold nanoparticles by sodium borohydride in the presence of mono-6-deoxy-6-pyridinium- $\beta$-cyclodextrin chloride," Journal of Physical Chemistry $C$, vol. 112, no. 2, pp. 443-451, 2008.

[19] C. Yu, H. Nakshatri, and J. Irudayaraj, "Identity profiling of cell surface markers by multiplex gold nanorod probes," Nano Letters, vol. 7, no. 8, pp. 2300-2306, 2007.

[20] M. Li, Y. Lin, C. Wu, and H. Liu, "Enhancing the efficiency of a PCR using gold nanoparticles," Nucleic Acids Research, vol. 33, no. 21, p. e184, 2005.

[21] X. M. Qian and S. M. Nie, "Single-molecule and single-nanoparticle SERS: from fundamental mechanisms to biomedical applications," Chemical Society Reviews, vol. 37, no. 5, pp. 912920, 2008.

[22] K. Lee and M. A. El-Sayed, "Gold and silver nanoparticles in sensing and imaging: sensitivity of plasmon response to size, shape, and metal composition," The Journal of Physical Chemistry B, vol. 110, no. 39, pp. 19220-19225, 2006.

[23] G. T. Wei, F. K. Liu, and C. R. C. Wang, "Shape separation of nanometer gold particles by size-exclusion chromatography," Analytical Chemistry, vol. 71, no. 11, pp. 2085-2091, 1999.

[24] F. K. Liu and G. T. Wei, "Effect of mobile-phase additives on separation of gold nanoparticles by size-exclusion chromatography," Chromatographia, vol. 59, no. 1-2, pp. 115-119, 2004.

[25] M. A. Uppal, A. Kafizas, T. H. Lim, and I. P. Parkin, "The extended time evolution size decrease of gold nanoparticles formed by the Turkevich method," New Journal of Chemistry, vol. 34, no. 7, pp. 1401-1407, 2010.

[26] W. Haiss, N. T. K. Thanh, J. Aveyard, and D. G. Fernig, "Determination of size and concentration of gold nanoparticles from UV-Vis spectra," Analytical Chemistry, vol. 79, no. 11, pp. 4215-4221, 2007.

[27] J. Kimling, M. Maier, B. Okenve, V. Kotaidis, H. Ballot, and A. Plech, "Turkevich method for gold nanoparticle synthesis revisited," The Journal of Physical Chemistry B, vol. 110, no. 32, pp. 15700-15707, 2006.

[28] B. Rodriguez-Gonzalez, P. Mulvaney, and L. M. Liz-Marzan, "An electrochemical model for gold colloid formation via citrate reduction," International Journal of Research in Physical Chemistry \& Chemical Physics, vol. 221, no. 3, pp. 415-426, 2007. 
[29] M. K. Chow and C. F. Zukoski, "Gold sol formation mechanisms: role of colloidal stability," Journal of Colloid And Interface Science, vol. 165, no. 1, pp. 97-109, 1994.

[30] B. L. V. Prasad, S. I. Stoeva, C. M. Sorensen, and K. J. Klabunde, "Digestive-ripening agents for gold nanoparticles: alternatives to thiols," Chemistry of Materials, vol. 15, no. 4, pp. 935-942, 2003.

[31] B. L. V. Prasad, S. I. Stoeva, C. M. Sorensen, and K. J. Klabunde, "Digestive ripening of thiolated gold nanoparticles: the effect of alkyl chain length," Langmuir, vol. 18, no. 20, pp. 7515-7520, 2002.

[32] Y. Dieckmann, H. Cölfen, H. Hofmann, and A. Petri-Fink, "Particle size distribution measurements of manganese-doped ZnS nanoparticles," Analytical Chemistry, vol. 81, no. 10, pp. 3889-3895, 2009.

[33] F. K. Liu, "Monitoring stability and sizes of Au/Pd core/shell nanoparticles by SEC," Chromatographia, vol. 70, no. 1-2, pp. 7-13, 2009.

[34] F. K. Liu and G. T. Wei, "Adding sodium dodecylsulfate to the running electrolyte enhances the separation of gold nanoparticles by capillary electrophoresis," Analytica Chimica Acta, vol. 510, no. 1, pp. 77-83, 2004.

[35] S. F. Sweeney, G. H. Woehrle, and J. E. Hutchison, "Rapid purification and size separation of gold nanoparticles via diafiltration," Journal of the American Chemical Society, vol. 128, no. 10, pp. 3190-3197, 2006.

[36] M. Hanauer, S. Pierrat, I. Zins, A. Lotz, and C. Sönnichsen, "Separation of nanoparticles by gel electrophoresis according to size and shape," Nano Letters, vol. 7, no. 9, pp. 2881-2885, 2007.

[37] F. K. Liu and Y. C. Chang, "Using size-exclusion chromatography to evaluate changes in the sizes of $\mathrm{Au}$ and $\mathrm{Au} / \mathrm{Pd}$ core/shell nanoparticles under thermal treatment," Chromatographia, vol. 74, no. 11-12, pp. 767-775, 2011.

[38] F. K. Liu, "SEC characterization of Au nanoparticles prepared through seed-assisted synthesis," Chromatographia, vol. 66, no. 9-10, pp. 791-796, 2007.

[39] F. K. Liu, "Monitoring the synthesis of Au nanoparticles using SEC," Chromatographia, vol. 68, no. 1-2, pp. 81-87, 2008.

[40] F. K. Liu, "Using size-exclusion chromatography to monitor the stabilization of $\mathrm{Au}$ nanoparticles in the presence of salt and organic solvent," Chromatographia, vol. 75, no. 19-20, pp. 1099-1105, 2012.

[41] F. K. Liu, P. W. Huang, Y. C. Chang, F. H. Ko, and T. C. Chu, "Microwave-assisted synthesis of silver nanorods," Journal of Materials Research, vol. 19, no. 2, pp. 469-473, 2004.

[42] F. K. Liu, "A high-efficiency capillary electrophoresis-based method for characterizing the sizes of Au nanoparticles," Journal of Chromatography A, vol. 1167, no. 2, pp. 231-235, 2007.

[43] S. Link and M. A. El-Sayed, "Size and temperature dependence of the plasmon absorption of colloidal gold nanoparticles," The Journal of Physical Chemistry B, vol. 103, no. 21, pp. 42124217, 1999.

[44] C. H. Kuo, T. F. Chiang, L. J. Chen, and M. H. Huang, "Synthesis of highly faceted pentagonal- And hexagonal-shaped gold nanoparticles with controlled sizes by sodium dodecyl sulfate," Langmuir, vol. 20, no. 18, pp. 7820-7824, 2004.

[45] Y. Y. Yu, S. S. Chang, C. L. Lee, and C. R. C. Wang, "Gold nanorods: electrochemical synthesis and optical properties," The Journal of Physical Chemistry B, vol. 101, no. 34, pp. 66616664, 1997.

[46] W. S. Wang, L. Zhen, C. Y. Xu, L. Yang, and W. Z. Shao, "Controlled synthesis of calcium tungstate hollow microspheres via ostwald ripening and their photoluminescence property,"
Journal of Physical Chemistry C, vol. 112, no. 49, pp. 1939019398, 2008.

[47] J. Liu, R. Xu, and A. E. Kaifer, "In situ modification of the surface of gold colloidal particles. Preparation of cyclodextrinbased rotaxanes supported on gold nanospheres," Langmuir, vol. 14, no. 26, pp. 7337-7339, 1998.

[48] J. Liu, S. Mendoza, E. Román, M. J. Lynn, R. Xu, and A. E. Kaifer, "Cyclodextrin-modified gold nanospheres. Host-guest interactions at work to control colloidal properties," Journal of the American Chemical Society, vol. 121, no. 17, pp. 4304-4305, 1999.

[49] J. Liu, W. Ong, E. Roman, M. J. Lynn, and A. E. Kaifer, "Cyclodextrin-modified gold nanospheres," Langmuir, vol. 16, no. 7, pp. 3000-3002, 2000.

[50] J. Alvarez, J. Liu, E. Román, and A. E. Kaifer, "Watersoluble platinum and palladium nanoparticles modified with thiolated $\beta$-cyclodextrin," Chemical Communications, no. 13, pp. 1151-1152, 2000.

[51] T. Zhu, X. Y. Fu, T. Mu, J. Wang, and Z. F. Liu, “pH-dependent adsorption of gold nanoparticles on p-aminothiophenolmodified gold substrates," Langmuir, vol. 15, no. 16, pp. 51975199, 1999.

[52] J. D. Senra, L. F. B. Malta, M. E. H. M. da Costa et al., "Hydroxypropyl- $\alpha$-cyclodextrin-capped palladium nanoparticles: active scaffolds for efficient carbon-carbon bond forming crosscouplings in water," Advanced Synthesis \& Catalysis, vol. 351, no. 14-15, pp. 2411-2422, 2009. 


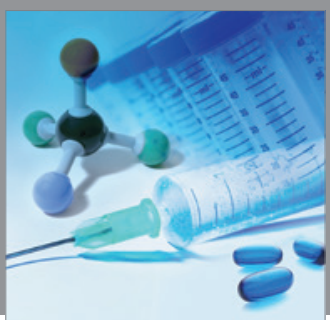

International Journal of

Medicinal Chemistry

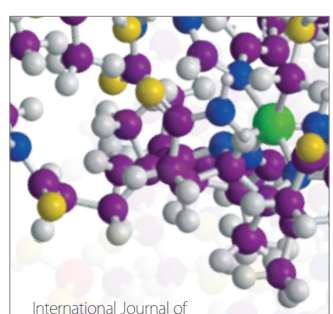

Carbohydrate Chemistry

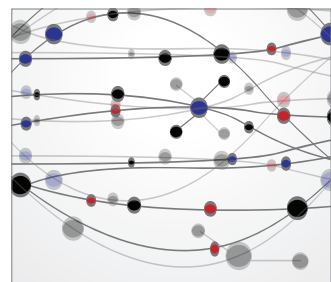

The Scientific World Journal
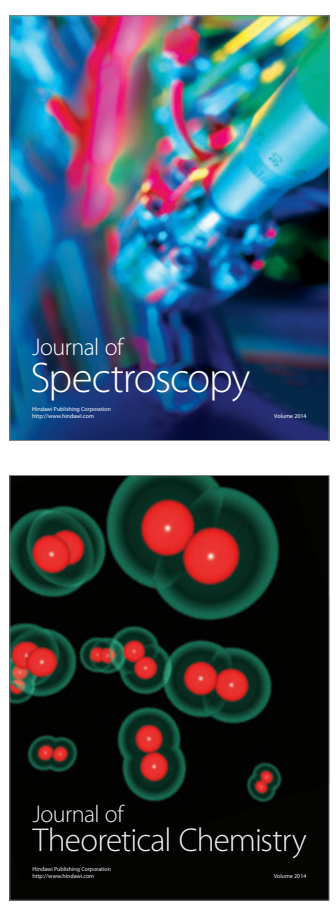
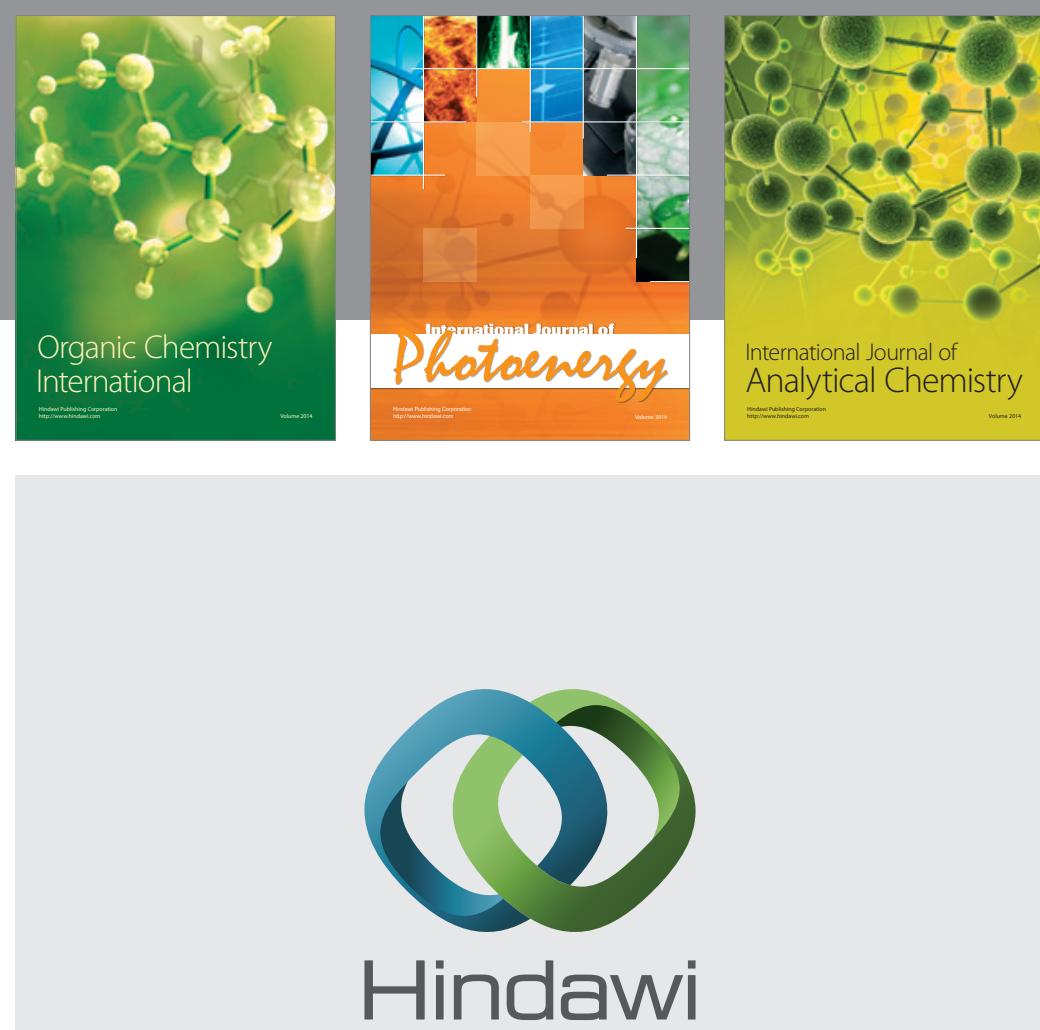

Submit your manuscripts at

http://www.hindawi.com
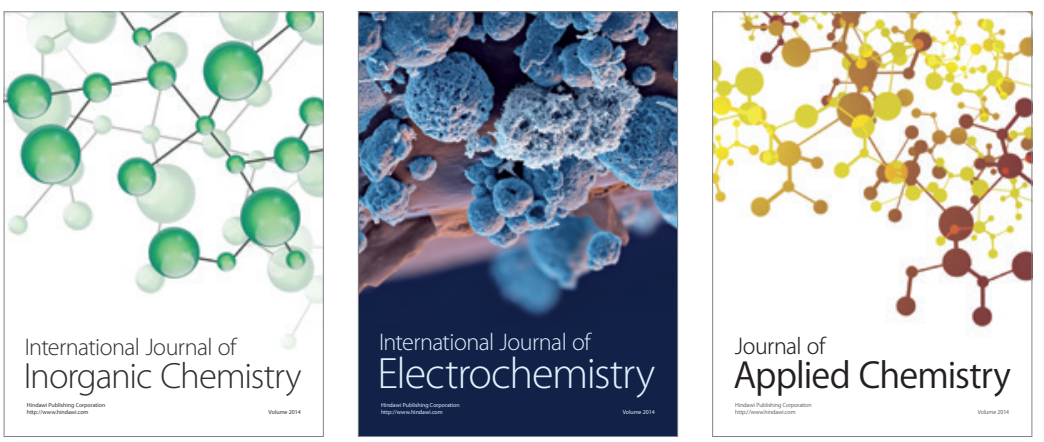

Journal of

Applied Chemistry
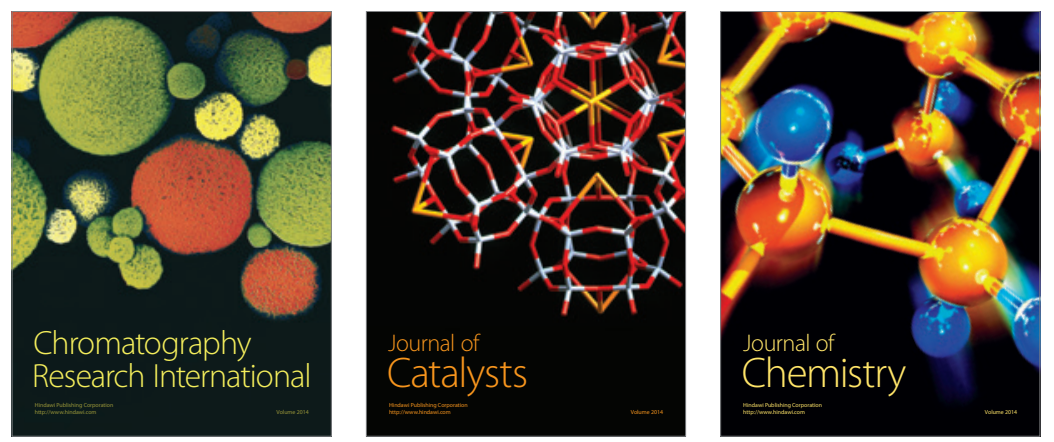
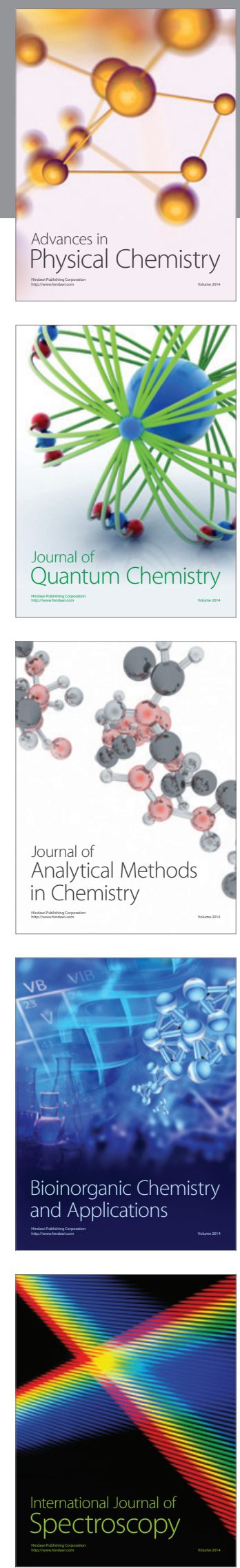